Supporting Information

\title{
Agglutination of Human Polyomaviruses by using a Tetravalent Glycocluster As a Cross-Linker
}

Makoto Ogata, ${ }^{1,2}, *$ Takashi Onoda, ${ }^{2}$ Ami Koizumi, ${ }^{2}$ Yuhei Tokunaga, ${ }^{3}$ Isao Ohta, ${ }^{3}$ Souichi Nukuzuma, ${ }^{4,5}$ Enoch Y. Park,${ }^{6}$ Taichi Usui, ${ }^{7}$ Tetsuro Suzuki ${ }^{8, *}$

${ }^{1}$ Faculty of Food and Agricultural Sciences, Fukushima University, 1 Kanayagawa, Fukushima city, Fukushima 960-1296, Japan

${ }^{2}$ Department of Applied Chemistry and Biochemistry, National Institute of Technology, Fukushima College, 30 Nagao, Iwaki, Fukushima 970-8034, Japan

${ }^{3}$ Advanced Research Facilities and Services, Preeminent Medical Photonics Education \& Research Center, Hamamatsu University School of Medicine, 1-20-1 Handayama, Higashi-ku, Hamamatsu 431-3192, Japan

${ }^{4}$ Department of Infectious Diseases, Kobe Institute of Health, 4-6-5, Minatojima, Nakamachi, Chuo-ku, Kobe 650-0046, Japan

${ }^{5}$ Present address: Kikuchi Research Center, KM Biologics Co., Ltd. 1314-1 Kyokushi Kawanabe, Kikuci-shi, Kumamoto 869-1298, Japan

${ }^{6}$ Research Institute of Green science and Technology, Shizuoka University, 836 Ohya, Surugaku, Shizuoka 422-8529, Japan

${ }^{7}$ Integrated Bioscience Research Division, Graduate School of Science and Technology, Shizuoka University, 836 Ohya, Suruga-ku, Shizuoka 422-8529, Japan

${ }^{8}$ Department of Virology and Parasitology, Hamamatsu University School of Medicine, 1-20-1 Handayama, Higashi-ku, Hamamatsu 431-3192, Japan

Corresponding Authors: ogata@agri.fukushima-u.ac.jp (M.O.), tesuzuki@hama-med.ac.jp (T.S.) 
Analytical Methods. Nuclear magnetic resonance (NMR) spectra were acquired by using a JEOL ECX-500 II spectrometer (Jeol Ltd., Tokyo, Japan). Mass spectra were acquired by using a JMS-T100LC mass spectrometer (Jeol Ltd., Tokyo, Japan) and a TripleTOF 5600 mass spectrometer (AB Sciex, Concord, ON, USA), respectively.

Ligands. (a) Compound 1. The monovalent sialo-ligand (1) was synthesized by enzymatically $\alpha 2,6$-sialylating the previously reported compound 5-TFAP- $\beta$-LacNAc ${ }^{1}$. In brief, a mixture containing $50.2 \mathrm{mg}$ of 5-TFAP- $\beta$-LacNAc, $25 \mathrm{mM} \mathrm{CMP- \beta -Neu5Ac,} 2.8 \mathrm{U} / \mathrm{mL}$ of a2,6-sialyltransferase from Photobacterium damsela (Cosmo Bio Co., Ltd, Tokyo, Japan), 2.5 $\mathrm{mM} \mathrm{MnCl}_{2}, 1.0 \mathrm{mg} / \mathrm{mL}$ of bovine serum albumin and $8.9 \mathrm{U} / \mathrm{mL}$ of calf intestine alkaline phosphatase (Boehringer-Mannheim GMBH, Mannheim, Germany) in 25 mM MOPS buffer $(\mathrm{pH} 7.4)$ in a total volume of $8.9 \mathrm{~mL}$ was incubated at $37^{\circ} \mathrm{C}$ for 30 hours. After heating to 100 ${ }^{\circ} \mathrm{C}$ and centrifugation $(8000 \times g, 10 \mathrm{~min})$, the supernatant from the reaction mixture was passed through an ODS column $(2.0 \times 30 \mathrm{~cm})$ equilibrated with $5 \%$ methanol at flow rate of $2.0 \mathrm{~mL} / \mathrm{min}$ and $20 \mathrm{~mL}$ fractions were collected. An aliquot from pooled fractions 9-13 was concentrated and lyophilized to yield compound $\mathbf{1}(76 \%, 58 \mathrm{mg}$ relative to the amount 5-TFAP- $\beta$-LacNAc). MS: $m / z$ 878.3034 [M+Na $]^{+}$(calcd for $\mathrm{C}_{32} \mathrm{H}_{52} \mathrm{~F}_{3} \mathrm{~N}_{3} \mathrm{NaO}_{20}$ : 878.2988); ${ }^{1} \mathrm{H} \mathrm{NMR}\left(\mathrm{D}_{2} \mathrm{O}, 500 \mathrm{MHz}\right.$, Supporting Information, Figure S1A): $\delta 4.55\left(\mathrm{~d}, 1 \mathrm{H}, J_{1,2} 7.0 \mathrm{~Hz}, \mathrm{H}-1\right), 4.44\left(\mathrm{~d}, 1 \mathrm{H}, J_{1^{\prime}, 2}, 8.0 \mathrm{~Hz}\right.$, H-1'), 4.00-3.52 (21H, from sugar, H- $\alpha), 3.32(\mathrm{t}, 2 \mathrm{H}, \mathrm{H}-\varepsilon), 2.63\left(\mathrm{dd}, 1 \mathrm{H}, J_{3}\right.$ "'ax,3"eq $12, J_{3}$ "eq,4" 3

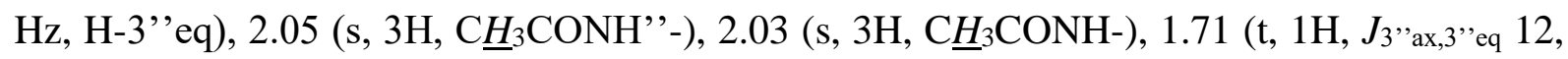

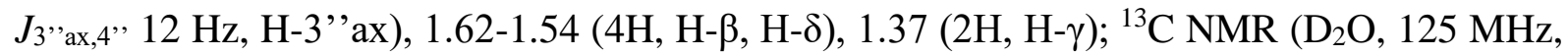
Supporting Information, Figure S1B): $\delta 177.7$ ( $\left.\mathrm{CH}_{3} \underline{\mathrm{CONH}}-\right), 177.2$ ( $\left.\mathrm{CH}_{3} \underline{\mathrm{CONH}}{ }^{\prime}{ }^{-}\right), 176.3$ (HOOC'”-), 161.6 ( $\left.\mathrm{CF}_{3} \underline{C O N H}-\right), 118.7$ ( $\left.\underline{C F}_{3} \mathrm{CONH}_{-}\right), 106.3$ (C-1'), 103.7 (C-1), 103.0 (C-2'’), 83.6 (C-4), 77.3 (C-5'), 76.5 (C-5), 75.4 (C-6’'), 75.3 (C-3), 75.2 (C-3'), 74.5 (C-8'’), 73.5 (C2'), 73.0 (C- $\alpha$ ), 71.2 (C-4', C-4'’), 71.0 (C-7'’), 66.1 (C-6'), 65.5 (C-9'’), 63.2 (C-6), 57.7 (C-

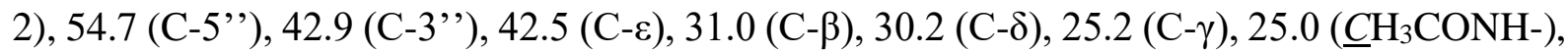




\section{$24.8\left(\underline{\mathrm{C}} \mathrm{H}_{3} \mathrm{CONH}^{\prime}{ }^{\prime}\right)$.}

(b) Compound 2. The bivalent sialo-ligand (2) was synthesized by mixing sialic acid and $11.2 \mathrm{mg}$ of bis-5-[5'-(3' '-thiobutanecarboxamido)pentanecarboxamido]pentyl- $\beta$ - $\mathrm{LacNAc}^{2}$ in a reaction similar to that used to prepare 1 and was obtained in a yield of $94 \%$ (15 mg). MS: $\mathrm{m} / \mathrm{z}$ $996.3876[\mathrm{M}+2 \mathrm{Na}]^{2+}, 1969.8224[\mathrm{M}+\mathrm{Na}]^{+}$(calcd for $\mathrm{C}_{80} \mathrm{H}_{138} \mathrm{~N}_{8} \mathrm{Na}_{2} \mathrm{O}_{42} \mathrm{~S}_{2}:$ 1992.8146, $\mathrm{C}_{80} \mathrm{H}_{138} \mathrm{~N}_{8} \mathrm{NaO}_{42} \mathrm{~S}_{2}:$ 1969.8248); ${ }^{1} \mathrm{H}$ NMR $\left(\mathrm{D}_{2} \mathrm{O}, 500 \mathrm{MHz}\right.$, Supporting Information, Figure S2A): $\delta 4.45\left(\mathrm{~d}, 2 \mathrm{H}, J_{1,2} 7.0 \mathrm{~Hz}, \mathrm{H}-1\right), 4.34\left(\mathrm{~d}, 2 \mathrm{H}, J_{1}, 2,8.0 \mathrm{~Hz}, \mathrm{H}-1\right.$ '), $3.91-3.40$ (42H, from sugar, H- $\alpha$ ), 3.07 (8H, H-e, H-e'), 2.63 (4H, H- $\alpha$ ), 2.57 (dd, 2H, $J_{3}$ "ax,3"'eq 12.5, J3"eq,4" $3.5 \mathrm{~Hz}$,

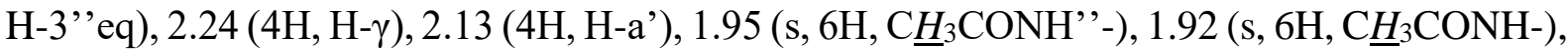
1.88 (4H, H- $\beta$ ), 1.61 (t, 2H, $J_{3}$ "ax,3"'eq 12.5, J3"'ax,4" 12.5 Hz, H-3'”ax), 1.53-1.36 (16H, H-b, Hd, H-b', H-d'), 1.22 (8H, H-c, H-c'); ${ }^{13} \mathrm{C}$ NMR ( $\mathrm{D}_{2} \mathrm{O}, 125 \mathrm{MHz}$, Supporting Information, Figure

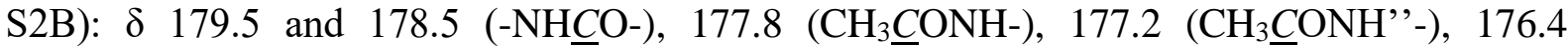
(HOOC'’), 106.3 (C-1'), 103.7 (C-1), 103.0 (C-2'’), 83.6 (C-4), 77.4 (C-5'), 76.5 (C-5), 75.4 (C-6’'), 75.4 (C-3), 75.3 (C-3'), 74.6 (C-8'”), 73.6 (C-2'), 73.1 (C-a), 71.3 (C-4', C-4'’), 71.1 (C-7'’), 66.2 (C-6'), 65.5 (C-9'’), 63.3 (C-6), 57.8 (C-2), 54.8 (C-5'’), 43.0 (C-3'’), 42.1 and 42.0 (C-e, C-e'), 40.0 (C- $\alpha$ ), 38.5 (C-a'), 37.2 (C- $\gamma$ ), 31.1 (C-b), 30.8 (C-d, C-d'), 28.4 (C-c'), 27.9 (C-b'), 27.7 (C- $\beta$ ), 25.4 (C-c), 25.2 ( $\left.\underline{\mathrm{C}} \mathrm{H}_{3} \mathrm{CONH}-\right), 24.9$ ( $\underline{\mathrm{C}} \mathrm{H}_{3} \mathrm{CONH}^{\prime \prime}$-).

(c) Compound 3. 2-(2-Aminoethoxy)ethyl- $\beta$-LacNAc (188 mg, $0.4 \mathrm{mmol})$ was coupled with EGTA (33.8 mg, $0.08 \mathrm{mmol}$ ) by our previously described protocols. ${ }^{3}$ Compound $\mathbf{3}$ was obtained in a total yield of $82 \%(143 \mathrm{mg})$ relative to the amount of EGTA. MALDI-TOF mass: $\mathrm{m} / \mathrm{z} 2189.8$ $[\mathrm{M}+\mathrm{H}]^{+}, 2211.8[\mathrm{M}+\mathrm{Na}]^{+}\left(\right.$calcd for $\left.\mathrm{C}_{86} \mathrm{H}_{153} \mathrm{~N}_{10} \mathrm{O}_{54}: 2190.0, \mathrm{C}_{86} \mathrm{H}_{152} \mathrm{~N}_{10} \mathrm{Na}_{1} \mathrm{O}_{54}: 2211.9\right) ;{ }^{1} \mathrm{H}$ NMR ( $\mathrm{D}_{2} \mathrm{O}, 500 \mathrm{MHz}$, Supporting Information, Figure S3): $\delta 4.58$ (d, 4H, $\left.J_{1,2} 8.0 \mathrm{~Hz}, \mathrm{H}-1\right)$, 4.48 (d, 4H, $\left.J_{1^{\prime}, 2}, 8.0 \mathrm{~Hz}, \mathrm{H}-1^{\prime}\right), 2.83$ (4H, H-b), 2.05 (s, 12H, $\left.\underline{H}_{3} \mathrm{CONH}-\right) ;{ }^{13} \mathrm{C}$ NMR $\left(\mathrm{D}_{2} \mathrm{O}\right.$,

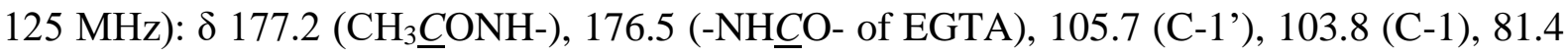
(C-4), 78.2 (C-5’), 77.6 (C-5), 75.4 (C-3’), 75.3 (C-3), 73.8 (C-2’), 72.4 (C-d), 72.3 (C- $\beta$ ), 71.8 


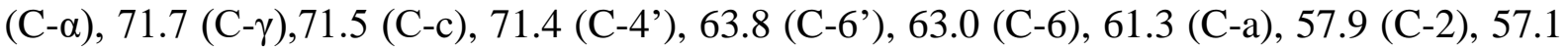
(C-b), $41.5(\mathrm{C}-\delta), 25.1\left(\underline{\mathrm{C}} \mathrm{H}_{3} \mathrm{CONH}-\right)$.

(d) Compound 4. 2-[2-(5'-Aminopentanecarboxamidoethoxy)]ethyl- $\beta$-LacNAc (175 mg, $0.3 \mathrm{mmol})$ was coupled with EGTA $(25 \mathrm{mg}, 0.067 \mathrm{mmol})$ by our previously described protocols. ${ }^{3}$ Compound 4 was obtained in a total yield of $82 \%$ (144 mg) relative to the amount of EGTA. MALDI-TOF mass: $m / z 2642.1[\mathrm{M}+\mathrm{H}]^{+}, 2664.1[\mathrm{M}+\mathrm{Na}]^{+}\left(\right.$calcd for $\mathrm{C}_{110} \mathrm{H}_{197} \mathrm{~N}_{14} \mathrm{O}_{58}$ : 2642.3, $\mathrm{C}_{110} \mathrm{H}_{196} \mathrm{~N}_{14} \mathrm{Na}_{1} \mathrm{O}_{58}$ : 2664.3); ${ }^{1} \mathrm{H} \mathrm{NMR}\left(\mathrm{D}_{2} \mathrm{O}, 500 \mathrm{MHz}\right.$, Supporting Information, Figure S4): $\delta 4.59$ (d, 4H, $\left.J_{1,2} 8.0 \mathrm{~Hz}, \mathrm{H}-1\right), 4.49$ (d, 4H, $\left.J_{1^{\prime}, 2}, 7.5 \mathrm{~Hz}, \mathrm{H}-1^{\prime}\right), 4.005$ (4H, H- $\left.\alpha \mathrm{b}\right), 4.004$ (dd, 4H, $\left.J_{5,6 \mathrm{~b}} 2.5, J_{6 \mathrm{a}, 6 \mathrm{~b}} 12.5 \mathrm{~Hz}, \mathrm{H}-6 \mathrm{~b}\right), 3.95$ (4H, H-4'), 3.85 (dd, 4H, $J_{5,6 \mathrm{a}} 5.5, J_{6 \mathrm{a}, 6 \mathrm{~b}} 12.5 \mathrm{~Hz}$, H-6a), 3.56 (dd, 4H, $\left.J_{1^{\prime}, 2^{\prime}}, 7.5, J_{2}^{\prime}, 3^{\prime} 10.0 \mathrm{~Hz}, \mathrm{H}-2^{\prime}\right), 3.38(8 \mathrm{H}, \mathrm{H}-\delta), 3.24\left(8 \mathrm{H}, \mathrm{H}-\varepsilon^{\prime}\right), 2.82(4 \mathrm{H}$, H-b), 2.27 (8H, H- $\left.\alpha^{\prime}\right), 2.05$ (s, 12H, $\left.\underline{\mathrm{H}}_{3} \mathrm{CONH}-\right), 1.62$ (8H, H- $\left.\beta^{\prime}\right) 1.55$ (8H, H- $\left.\delta^{\prime}\right), 1.33$ (8H, $\left.\mathrm{H}-\gamma^{\prime}\right) ;{ }^{13} \mathrm{C}$ NMR $\left(\mathrm{D}_{2} \mathrm{O}, 125 \mathrm{MHz}\right): \delta 179.6$ (-NHCO- of spacer), $177.2\left(\mathrm{CH}_{3} \underline{C O N H}-\right), 176.0(-$ NH므- of EGTA), 105.7 (C-1'), 103.8 (C-1), 81.4 (C-4), 78.2 (C-5'), 77.6 (C-5), 75.4 (C-3’) 75.3 (C-3), 73.8 (C-2’), 72.5 (C-d), 72.3 (C- $\beta$ ), 71.8 (C- $\alpha$, C- $\gamma$, C-c), 71.4 (C-4'), 63.8 (C-6’), 62.9 (C-6), 61.5 (C-a), 57.9 (C-2), 57.5 (C-b), 41.8 (C- $\left.\varepsilon^{\prime}\right), 41.7$ (C- $\delta$ ), $38.4\left(\mathrm{C}-\alpha^{\prime}\right), 31.1$ (C- $\left.\delta^{\prime}\right)$, 28.5 (C- $\left.\gamma^{\prime}\right), 27.8$ (C- $\beta$ '), $25.1\left(\underline{\mathrm{C}} \mathrm{H}_{3} \mathrm{CONH}-\right)$.

(e) Compound 5. The tetravalent sialo-ligand (5) was synthesized by mixing sialic acid and $22 \mathrm{mg}$ of compound $\mathbf{3}$ by our previously described protocols. ${ }^{3}$ Compound $\mathbf{5}$ was obtained in a yield of $68 \%$ (23 mg). MALDI-TOF mass: $\mathrm{m} / z 3354.3[\mathrm{M}+\mathrm{H}]^{+}, 3376.3[\mathrm{M}+\mathrm{Na}]^{+}$(calcd for $\left.\mathrm{C}_{130} \mathrm{H}_{221} \mathrm{~N}_{14} \mathrm{O}_{86}: 3354.3, \mathrm{C}_{130} \mathrm{H}_{220} \mathrm{~N}_{14} \mathrm{Na}_{1} \mathrm{O}_{86}: 3376.3\right) ;{ }^{1} \mathrm{H}$ NMR $\left(\mathrm{D}_{2} \mathrm{O}, 500 \mathrm{MHz}\right.$, Supporting Information, Figure S5): $\delta 4.61$ (4H, H-1), 4.46 (d, 4H, $J_{1}{ }^{\prime}, 2^{\prime}, 8.0 \mathrm{~Hz}, \mathrm{H}-1^{\prime}$ ), 2.85 (4H, H-b), 2.69

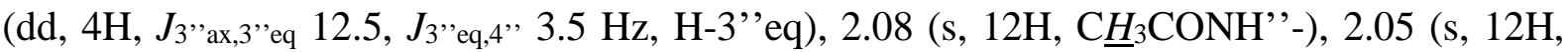

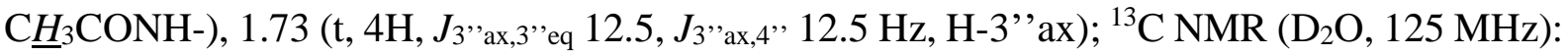

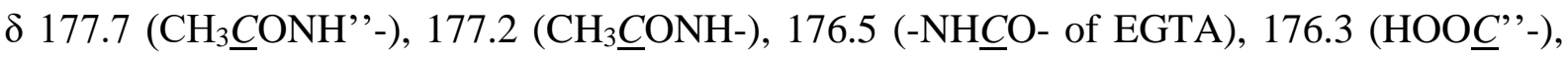
106.3 (C-1'), 103.7 (C-1), 103.0 (C-2'’), 83.6 (C-4), 77.3 (C-5'), 76.5 (C-5), 75.40 (C-6’), 75.37 
(C-3), 75.28 (C-3’), 74.5 (C-8’’), 73.6 (C-2'), 72.3 (C- $\beta$, C-d), 71.8 (C- $\alpha), 71.7$ (C- $\gamma), 71.5$ (Cc), 71.3 and 71.2 (C-4'”, C-4'), 71.0 (C-7'’), 66.2 (C-6'), 65.5 (C-9'’), 63.2 (C-6), 61.4 (C-a),

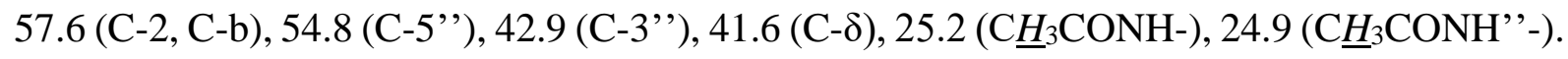

(f) Compound 6. The tetravalent sialo-ligand (6) was synthesized by mixing sialic acid and $26 \mathrm{mg}$ of compound 4 by our previously described protocols. ${ }^{3}$ Compound 6 was obtained in a yield of 63\% (24 mg). MALDI-TOF mass: $\mathrm{m} / z 3806.9[\mathrm{M}+\mathrm{H}]^{+}, 3828.9[\mathrm{M}+\mathrm{Na}]^{+}($calcd for $\mathrm{C}_{154} \mathrm{H}_{265} \mathrm{~N}_{18} \mathrm{O}_{90}$ : 3806.7, $\left.\mathrm{C}_{154} \mathrm{H}_{264} \mathrm{~N}_{18} \mathrm{Na}_{1} \mathrm{O}_{90}: 3828.7\right) ;{ }^{1} \mathrm{H}$ NMR ( $\mathrm{D}_{2} \mathrm{O}, 500 \mathrm{MHz}$, Supporting Information, Figure S6): $\delta 4.61$ (d, $\left.4 \mathrm{H}, J_{1,2} 8.0 \mathrm{~Hz}, \mathrm{H}-1\right), 4.46\left(\mathrm{~d}, 4 \mathrm{H}, J_{1^{\prime}, 2}, 8.0 \mathrm{~Hz}, \mathrm{H}-1^{\prime}\right.$ ) $), 3.38$

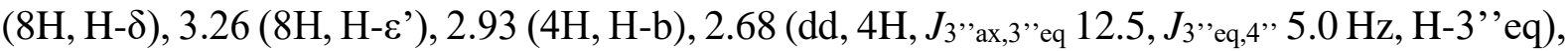

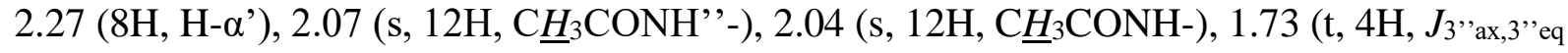

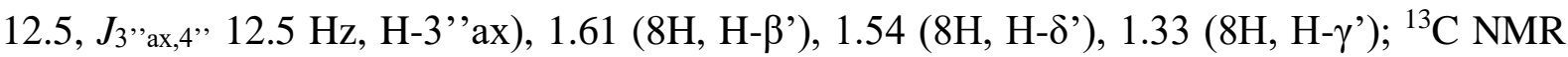
$\left(\mathrm{D}_{2} \mathrm{O}, 125 \mathrm{MHz}\right): \delta 179.6$ (-NH$\underline{C O}-$ of spacer), $177.7\left(\mathrm{CH}_{3} \underline{\mathrm{CONH}}{ }^{\prime}-\right), 177.2\left(\mathrm{CH}_{3} \underline{\mathrm{CONH}}-\right)$, 176.4 (HOO $\underline{C}$ '”-), 176.2 (-NH므- of EGTA), 106.3 (C-1'), 103.7 (C-1), 103.0 (C-2'’), 83.6 (C4), 77.3 (C-5'), 76.5 (C-5), 75.4 (C-6', C-3) 75.3 (C-3'), 74.5 (C-8'’), 73.6 (C-2'), 72.3 (C- $\beta$ ),

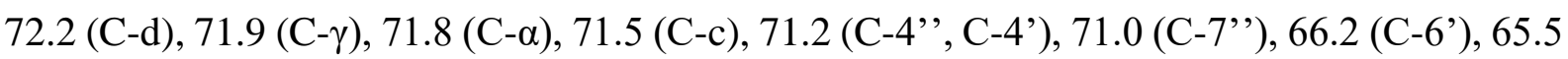
(C-9'’), 63.2 (C-6), 62.0 (C-a), 59.4 (C-b), 57.6 (C-2), 54.8 (C-5'’), 42.9 (C-3’'), 42.2 (C- $\delta$ ), 41.8 (C- $\left.\varepsilon^{\prime}\right), 38.4$ (C- $\left.\alpha^{\prime}\right), 30.8$ (C- $\left.\delta^{\prime}\right), 28.4$ (C- $\left.\gamma^{\prime}\right), 27.8$ (C- $\left.\beta^{\prime}\right), 25.2\left(\underline{C} \mathrm{H}_{3} \mathrm{CONH}-\right), 24.9$ $\left(\underline{\mathrm{C}} \mathrm{H}_{3} \mathrm{CONH}{ }^{\prime},-\right)$. 

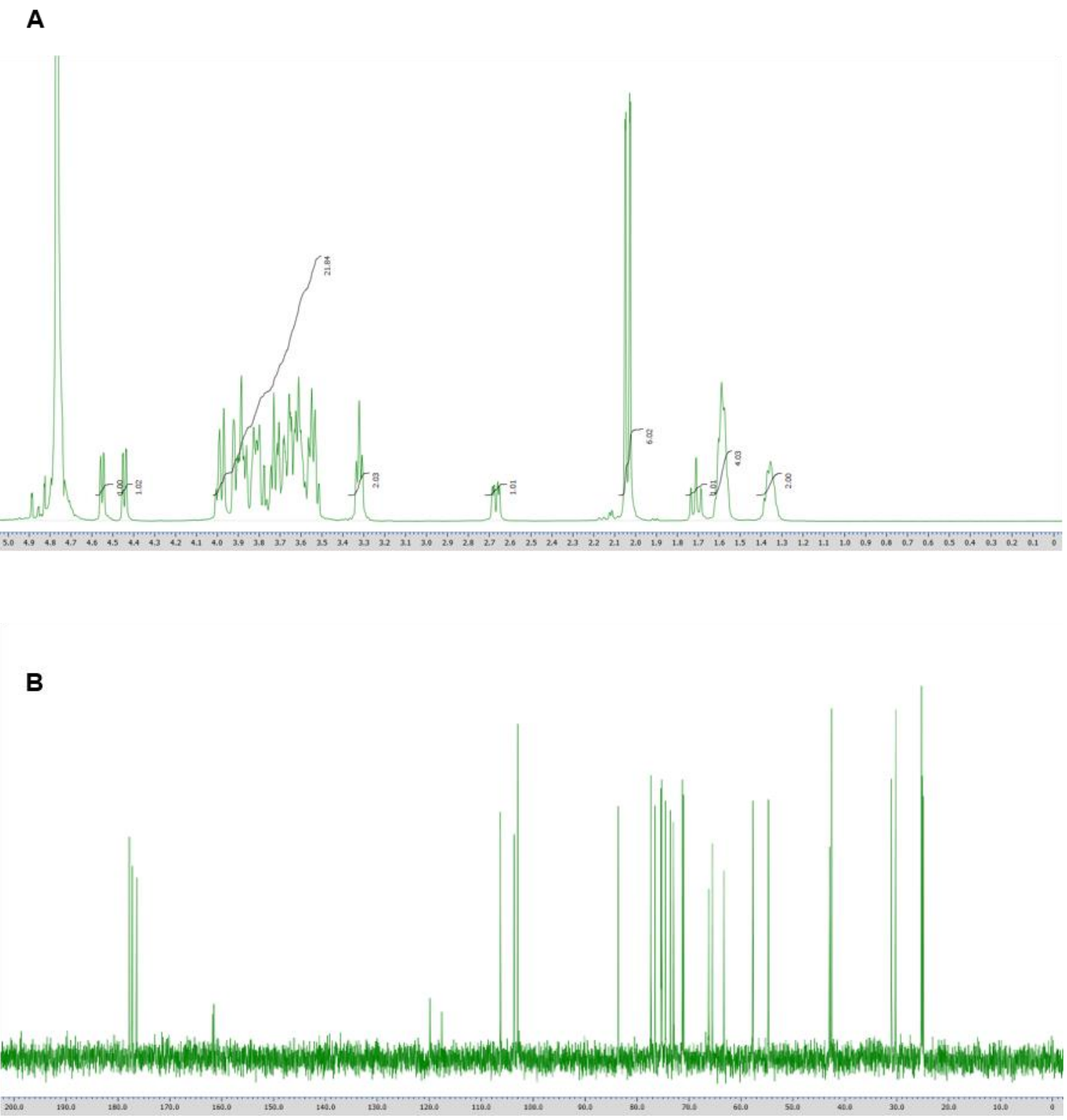

Figure S1. (A) $500-\mathrm{MHz}{ }^{1} \mathrm{H}$ NMR spectrum/integral value and (B) $125-\mathrm{MHz}{ }^{13} \mathrm{C}$ NMR spectrum of ligand $\mathbf{1}$. Solvent, $\mathrm{D}_{2} \mathrm{O}$; temperature, $25^{\circ} \mathrm{C}$; concentration, $5 \mathrm{mg} / 600 \mu \mathrm{L}$. 
A

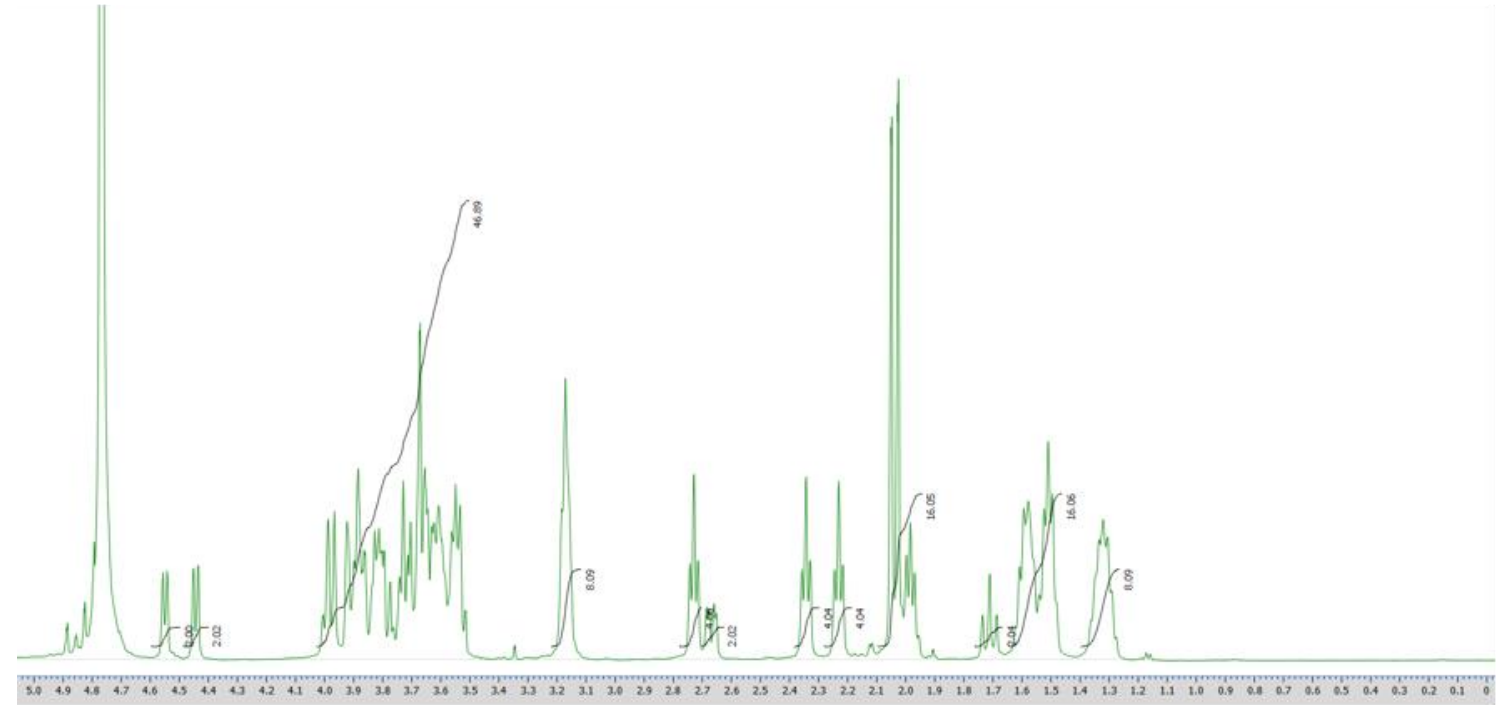

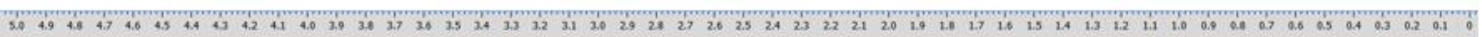

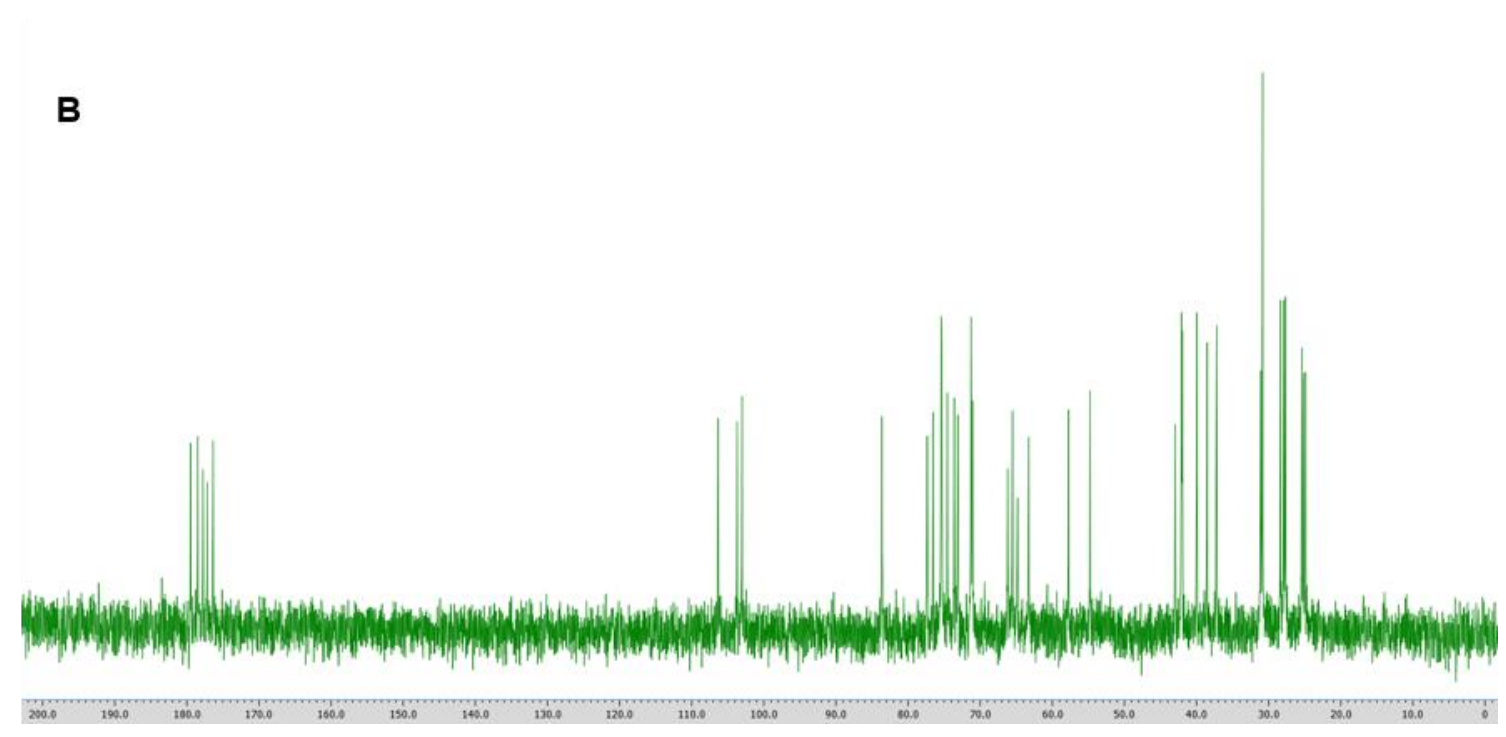

Figure S2. (A) $500-\mathrm{MHz}{ }^{1} \mathrm{H}$ NMR spectrum/integral value and (B) $125-\mathrm{MHz}{ }^{13} \mathrm{C}$ NMR spectrum of ligand 2. Solvent, $\mathrm{D}_{2} \mathrm{O}$; temperature, $25^{\circ} \mathrm{C}$; concentration, $5 \mathrm{mg} / 600 \mu \mathrm{L}$. 


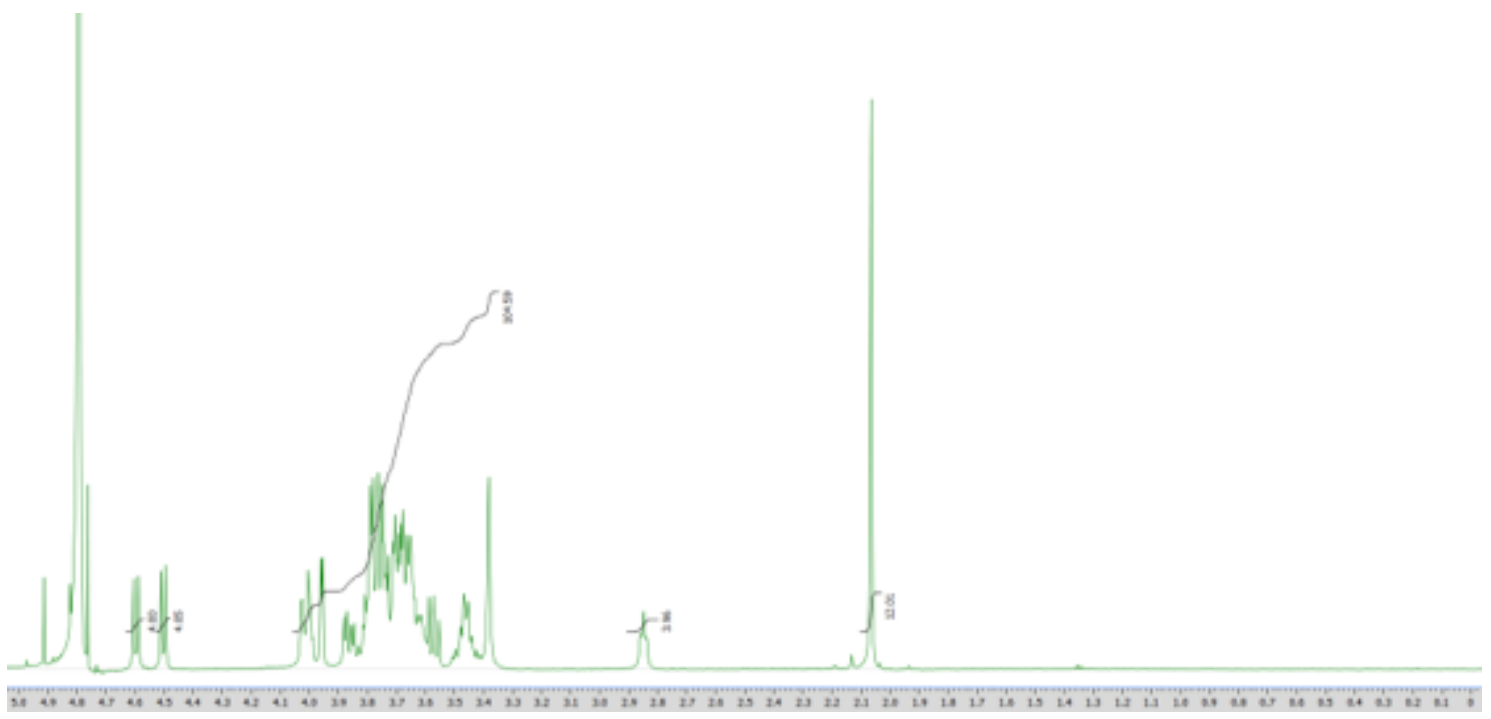

Figure S3. $500-\mathrm{MHz}{ }^{1} \mathrm{H}$ NMR spectrum and integral value of ligand 3. Solvent, $\mathrm{D}_{2} \mathrm{O}$; temperature, $25^{\circ} \mathrm{C}$; concentration, $5 \mathrm{mg} / 600 \mu \mathrm{L}$. 


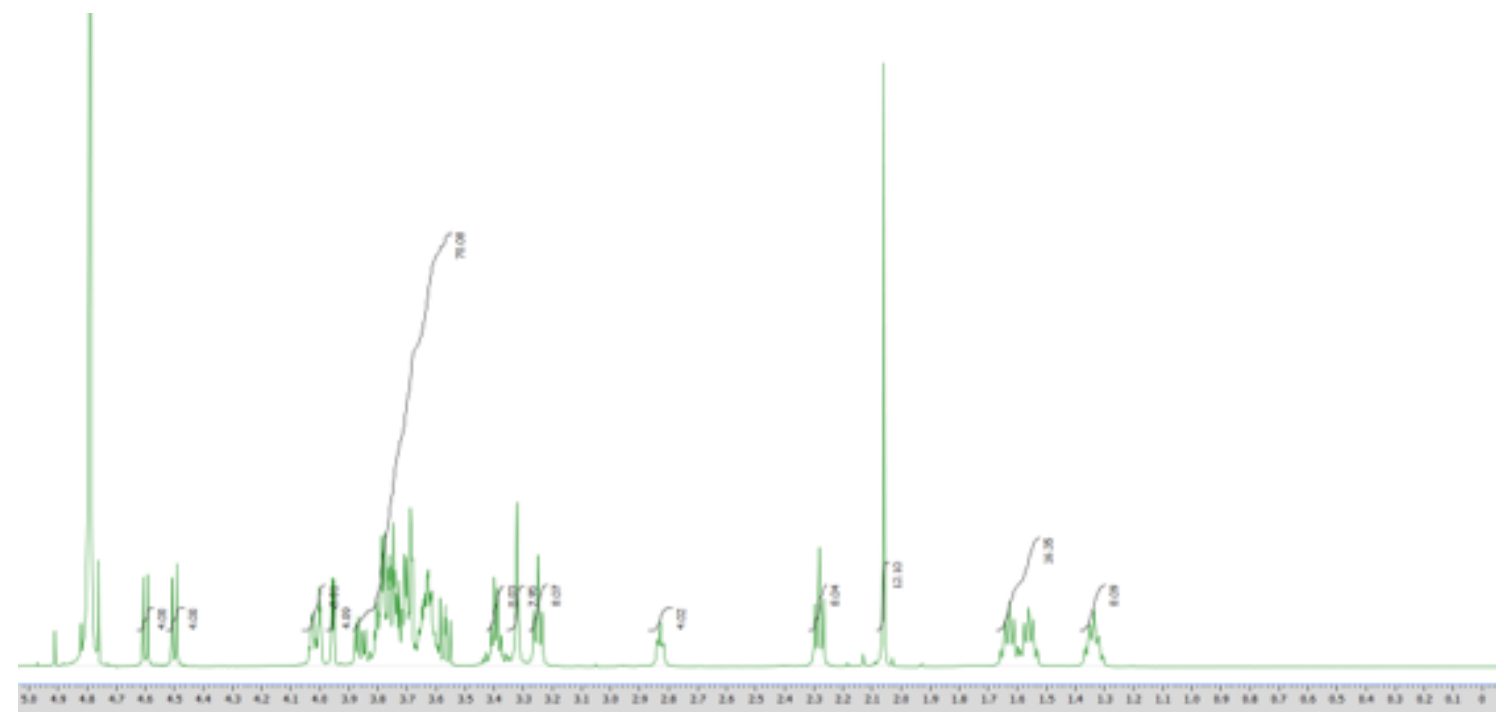

Figure S4. $500-\mathrm{MHz}{ }^{1} \mathrm{H}$ NMR spectrum and integral value of ligand 4. Solvent, $\mathrm{D}_{2} \mathrm{O}$; temperature, $25^{\circ} \mathrm{C}$; concentration, $5 \mathrm{mg} / 600 \mu \mathrm{L}$. 


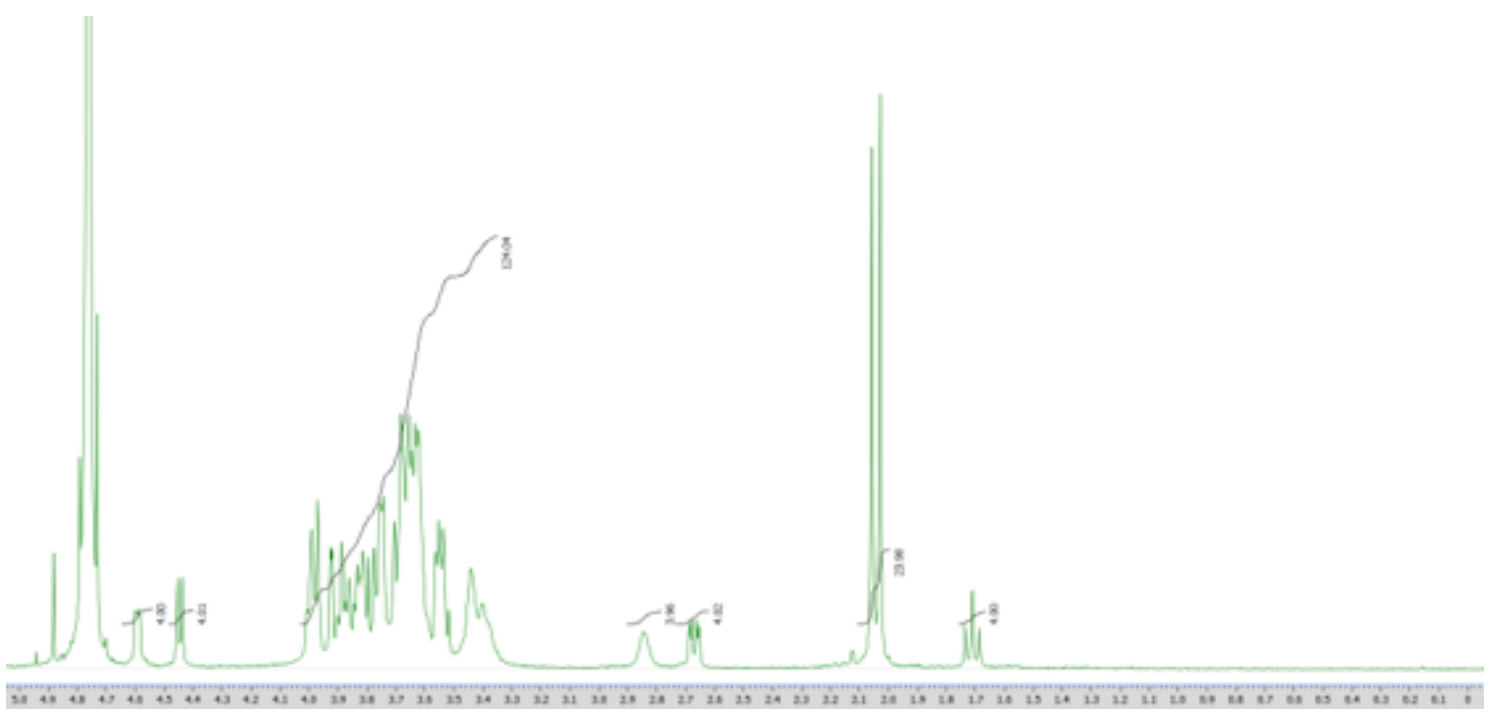

Figure S5. 500-MHz ${ }^{1} \mathrm{H}$ NMR spectrum and integral value of ligand 5. Solvent, $\mathrm{D}_{2} \mathrm{O}$; temperature, $25^{\circ} \mathrm{C}$; concentration, $5 \mathrm{mg} / 600 \mu \mathrm{L}$. 


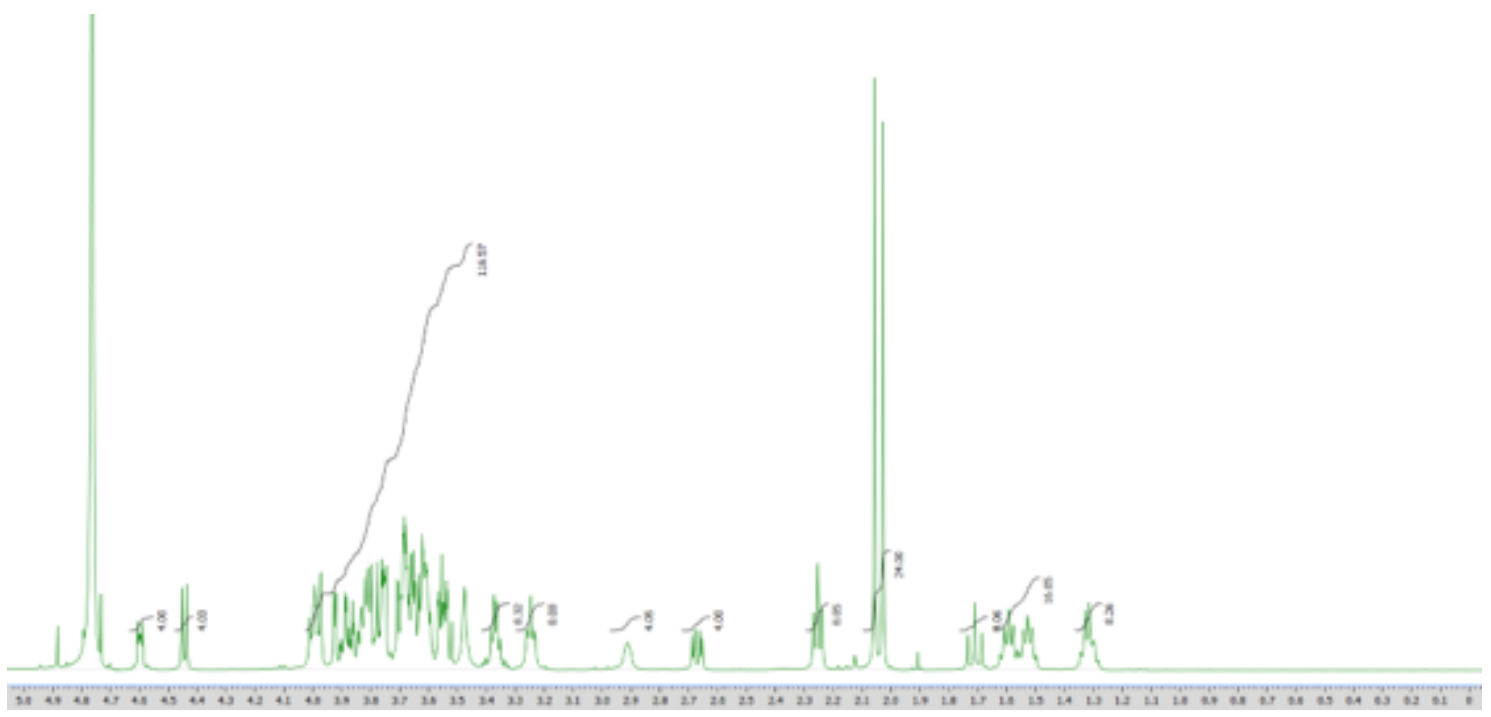

Figure S6. $500-\mathrm{MHz}{ }^{1} \mathrm{H}$ NMR spectrum and integral value of ligand 6. Solvent, $\mathrm{D}_{2} \mathrm{O}$; temperature, $25^{\circ} \mathrm{C}$; concentration, $5 \mathrm{mg} / 600 \mu \mathrm{L}$. 
A
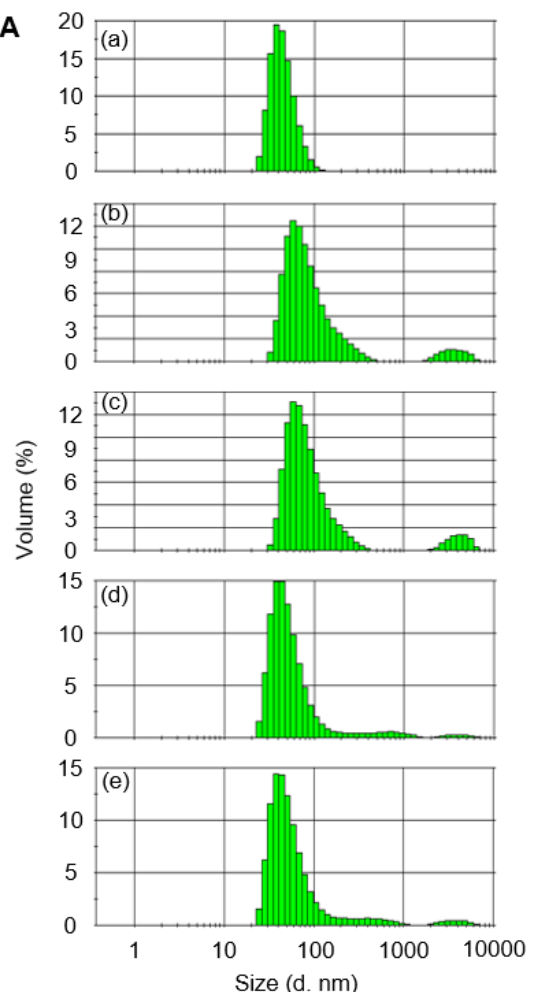

C
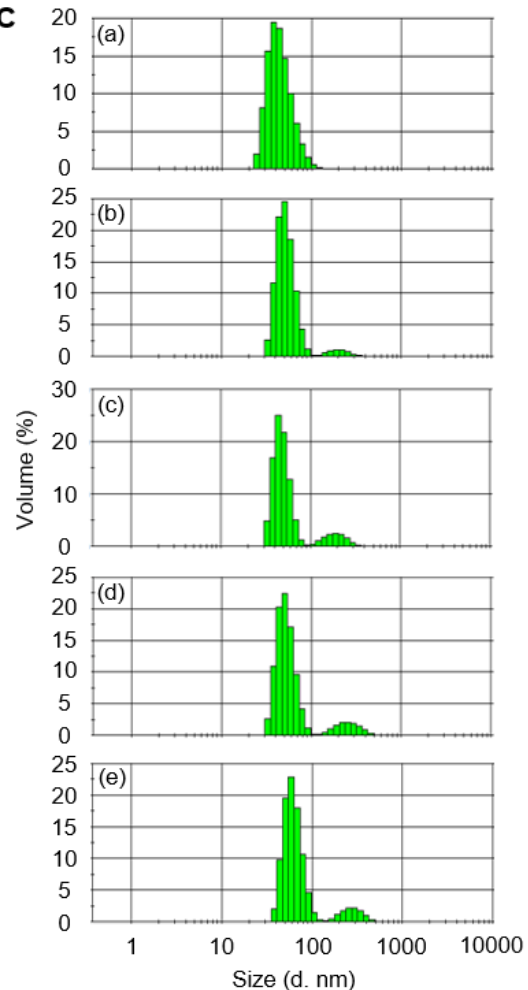
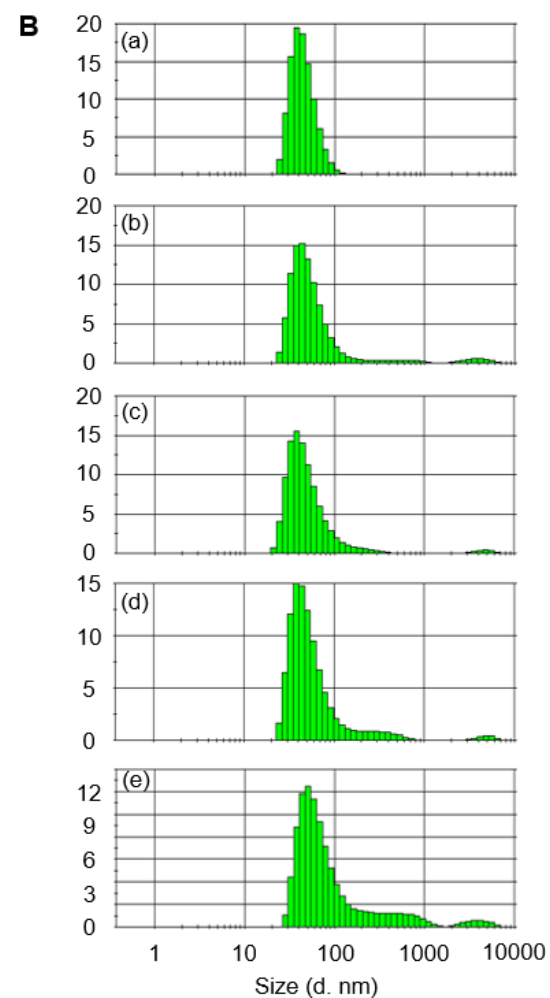

D
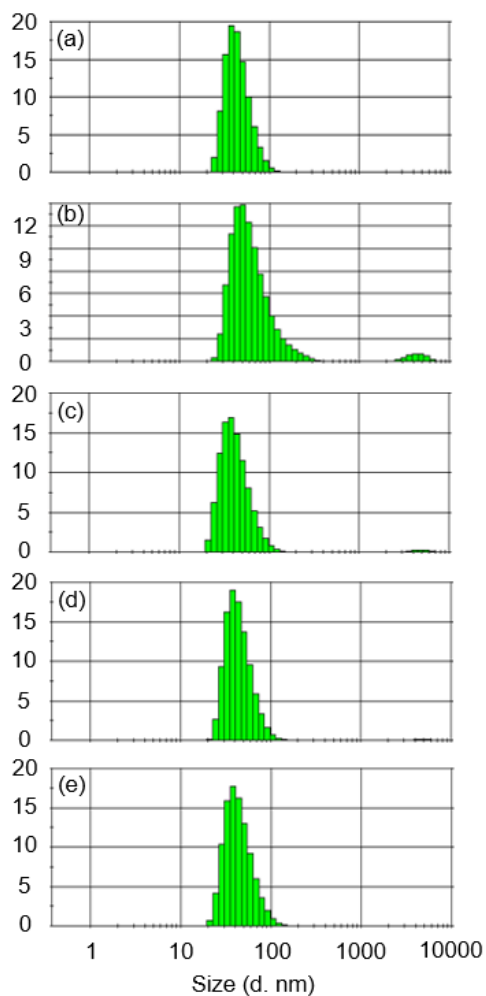

Figure S7. DLS measurements of the complexes formed by 1-4 and MCPyV-LP. MCPyV-LP solution $(0.01 \mathrm{mg} / \mathrm{mL})$ was incubated with increasing concentrations of $1(A), 2(B), 3(C)$, or 4 (D) in $10 \mathrm{mM}$ PBS (pH 7.4): (a) $0 \mathrm{mM}$, (b) $0.031 \mathrm{mM}$, (c) $0.063 \mathrm{mM}$, (d) $0.25 \mathrm{mM}$, and (e) 1.0 $\mathrm{mM}$. After each addition of ligand, the samples were incubated for $30 \mathrm{~min}$ at $25{ }^{\circ} \mathrm{C}$. DLS intensities were measured at $25{ }^{\circ} \mathrm{C}$ by using a Zetasizer Nano ZSP instrument. The size distribution of the particles is reported relative to the scattering volume. 

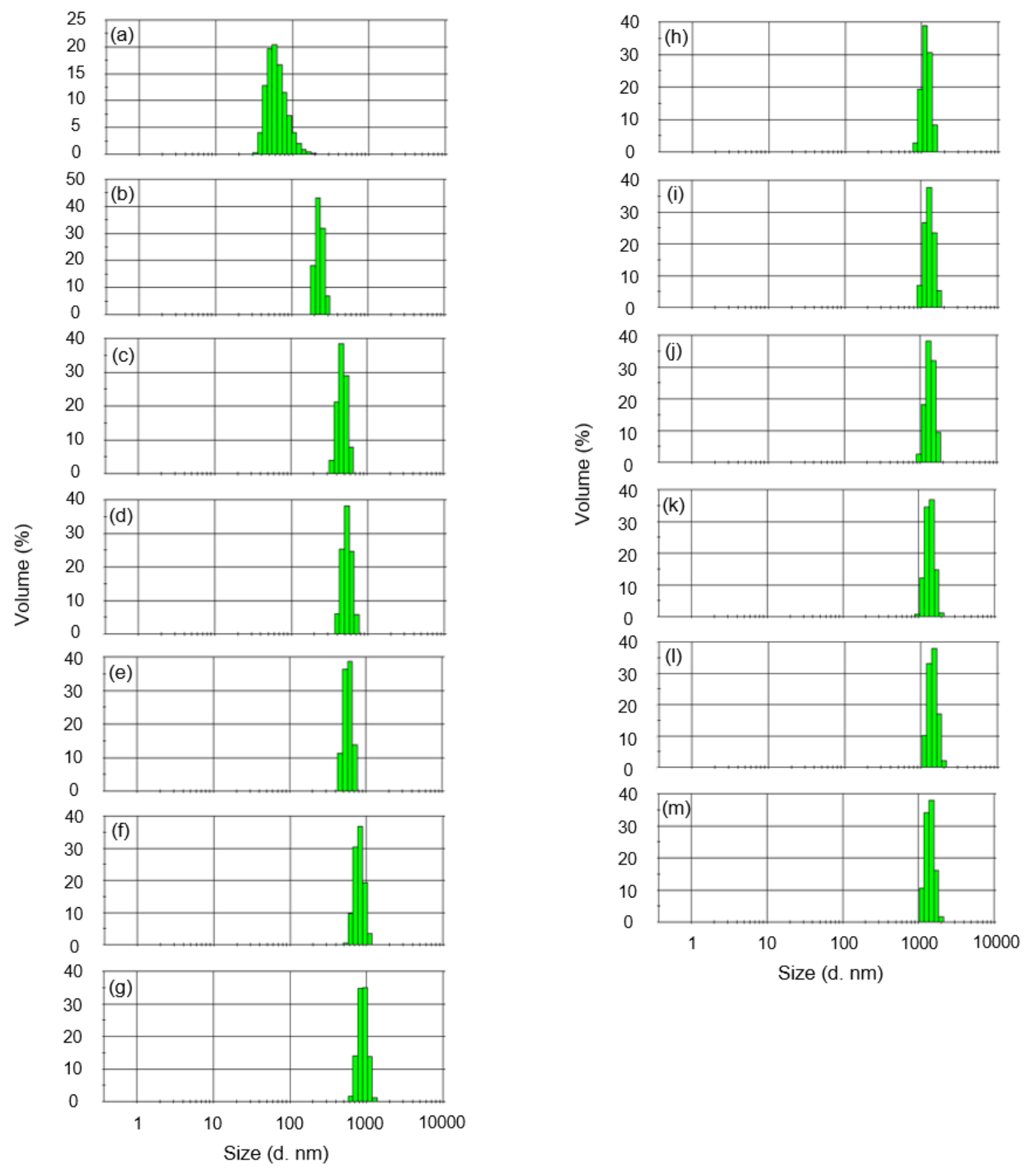

Figure S8. DLS measurements of the complexes formed by 5 and MCPyV-LP. MCPyV-LP solution $(0.01 \mathrm{mg} / \mathrm{mL})$ was mixed with increasing concentrations of ligand $5(0.25 \mathrm{mM})$ at 25 ${ }^{\circ} \mathrm{C}$ in $10 \mathrm{mM}$ PBS (pH 7.4): (a) $0 \mathrm{~s}$, (b) $2 \mathrm{~s}$, (c) $38 \mathrm{~s}$, (d) $1 \min 15 \mathrm{~s}$, (e) $2 \mathrm{~min}$, (f) $2 \mathrm{~min} 40 \mathrm{~s}$, (g) $3 \mathrm{~min} 20 \mathrm{~s}$, (h) $4 \mathrm{~min} 20 \mathrm{~s}$, (i) $5 \mathrm{~min}$, (j) $5 \mathrm{~min} 40 \mathrm{~s}$, (k) $7 \operatorname{min~} 10 \mathrm{~s}$, (l) $8 \mathrm{~min}$, and (m) 8 min 50 s. DLS intensities were measured at $25^{\circ} \mathrm{C}$ by using a Zetasizer Nano ZSP. The size distribution of the particles is reported relative to the scattering volume. 


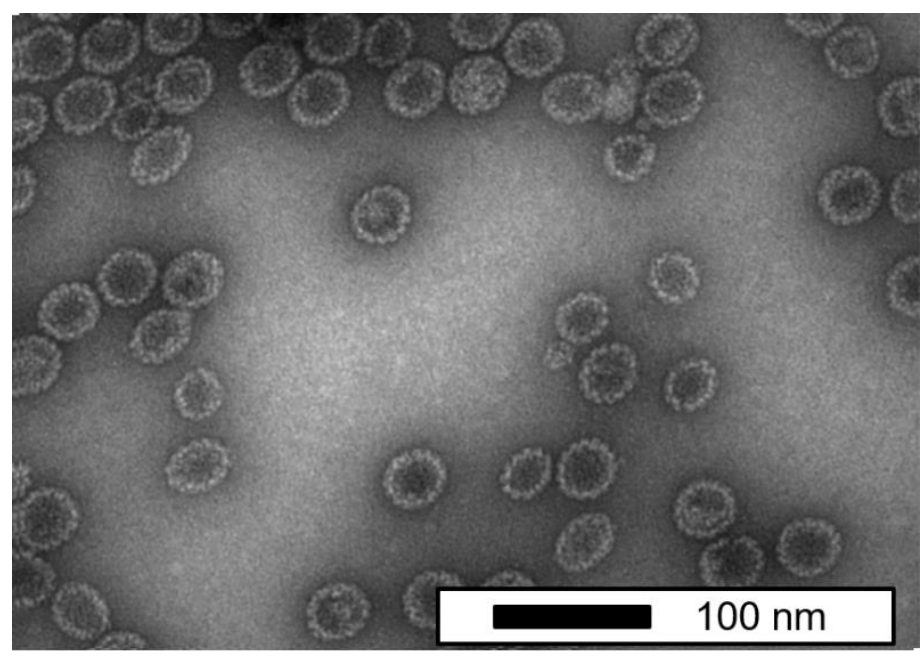

Figure S9. TEM image of MCPyV-LP particles $(0.01 \mathrm{mg} / \mathrm{mL})$ negatively strained with $2 \%$ uranyl acetate. Bar, $100 \mathrm{~nm}$. 


\section{References}

(1) Ogata, M.; Murata, T.; Murakami, K.; Suzuki, T.; Hidari, K. I. P. J.; Suzuki, Y.; Usui, T. Chemoenzymatic synthesis of artificial glycopolypeptides containing multivalent sialyloligosaccharides with a $\gamma$-polyglutamic acid backbone and their effect on inhibition of infection by influenza viruses. Bioorg. Med. Chem. 2007, 15, 1383-1393.

(2) Endo, T.; Matsuda, S.; Obara, T.; Chuma, Y.; Ogata, M.; Yanagida, Y.; Hatsuzawa, T.; Usui, T. Label-free detection of oligosaccharide-lectin interaction using plasmonic optical device for glycomics application. Sens. Mater. 2011, 23, 135-146.

(3) Ogata, M.; Yano, M.; Umemura, S.; Murata, T.; Park, E. Y.; Kobayashi, Y.; Asai, T.; Oku, N.; Nakamura, N.; Matsuo, I.; Usui, T. Design and synthesis of high-avidity tetravalent glycoclusters as probes for Sambucus sieboldiana agglutinin and characterization of their binding properties. Bioconjugate Chem. 2012, 23, 97-105. 